\title{
Proximity Detection - Schutzeinrichtung für Gefahrenzonen im Untertageberg- und Tunnelbau: Stand der Technik und Notwendigkeiten der Weiterentwicklung
}

\author{
Nikolaus August Sifferlinger \\ Lehrstuhl f. Bergbaukunde, Bergtechnik u. Bergwirtschaft, Montanuniversität Leoben, Leoben, \\ Österreich
}

Eingegangen am 21. Dezember 2015; angenommen am 29. Dezember 2015; online publiziert am 9. Februar 2016

Zusammenfassung: Die räumliche Beengtheit in untertägigen Arbeitsstätten in Berg- undTunnelbau führt immer wieder zur Gefahr von Kollisionen zwischen Mensch und Maschine. Trotz entsprechender Arbeitsvorschriften und Sicherheitsschulungen sind Unfälle dieser Art immer noch zahlreich, und es wurden daher in den letzten Jahren technische Sicherheitseinrichtungen gefordert und entwickelt, die solche Unfälle vermeiden helfen. In den Vereinigen Staaten von Amerika und in Südafrika sind eine rasch steigende Anzahl von Proximity Detection Systemen im Einsatz, und damit ist eine erste Bewertung der Wirkung auf die Sicherheit der Bergleute möglich. Dieser Artikel fasst den Stand der Technik zusammen und zeigt aus den bisherigen Einsatzerfahrungen auch die Notwendigkeiten der Weiterentwicklung auf diesem Gebiet.

Schlüsselwörter: Gefahrenzone, Schutzeinrichtung, Untertage, Sicherheit, Maschinen, Unfallvermeidung

Proximity Detection in No-Go-Zones-A Safety Device for Underground Mining and Tunnelling: State of the Art and Necessary Further Developments

Abstract: The restricted space in underground work sites in mining and tunnelling always entails the potential of collisions between men and machinery. Despite work rules and safety training, accidents of this category are still too frequent and therefore technical safety systems to avoid such collisions have been asked for and developed. In the United States of America and South Africa a rising number of such proximity detection systems are in

DI. Dr. mont. N. A. Sifferlinger $(\triangle)$

Lehrstuhl f. Bergbaukunde, Bergtechnik u. Bergwirtschaft,

Montanuniversität Leoben,

Franz-Josef-Str. 18,

8700 Leoben, Österreich

E-Mail: nikolaus-august.sifferlinger@unileoben.ac.at operation and allow a first review of the impact on safety of miners. This article presents the state of the art and based on the existing operational experience some of the necessary further developments in this field.

Keywords: Proximity Detection, No-go-zone, Underground, Safety, Machinery, Accident avoidance

\section{Einleitung}

Die räumliche Beengtheit in untertägigen Arbeitsstätten in Berg- und Tunnelbau führt immer wieder zur Gefahr von Kollisionen zwischen Mensch und Maschine. Trotz entsprechender Arbeitsvorschriften und Sicherheitsschulungen sind Unfälle dieser Art immer noch zahlreich, und es wurden daher in den letzten Jahren technische Sicherheitseinrichtungen gefordert und entwickelt, die solche Unfälle vermeiden helfen.

\section{Hintergrund und Unfallzahlen}

Im Obertagebau ist es besonders die Gefahr von Kollisionen von Fahrzeugen untereinander, die sich im toten Sichtwinkel eines Fahrers befinden, die bei Unfällen zu schweren oder tödlichen Verletzungen der Passagiere führen. Die Benutzung der Fahrwege durch Fußgänger ist hier aus Sicherheitsgründen verboten. Die technischen Sicherheitseinrichtungen im Obertagebergbau konzentrieren sich daher auf die Vermeidung von Kollisionen durch "Collision Avoidance Systems".

Dieser Artikel befasst sich mit den Sicherheitseinrichtungen für den Untertagebereich. Dabei geht es um den Schutz von Personen im Gefahrenbereich in der Nähe von Maschinen. Die Systeme werden in erster Linie zur „Proximity Detection of persons in danger zones", also dem 


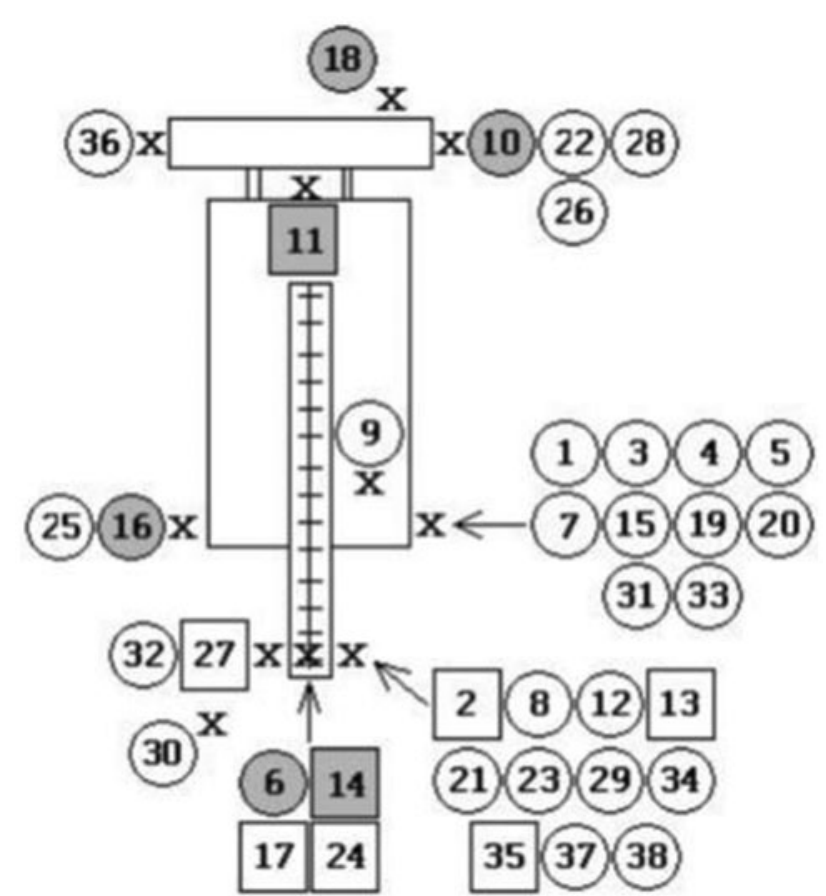

Abb. 1: Orte der 38 tödlichen Unfälle an funkferngesteuerten Continuous Minern im US-Kohlenbergbau im Zeitraum 1984 bis 2014. Die Schneidtrommel ist oben, der Förderer am Heck der Maschine. Die Nummern beziehen sich auf die einzelnen Unfälle in der angeführten MSHA-Analyse. Das " $x^{\prime \prime}$ kennzeichnet den ungefähren Unfallort. Eine graue Hinterlegung zeigt, dass der Unfall während der Wartung geschah. Ein Kreis um die Nummer zeigt, dass das Opfer der Maschinenfahrer war, ein Quadrat bezeichnet ein Opfer, das als Helfer in der Sektion arbeitete. (Quelle: MSHA [1])

Erkennen von Personen in Gefahrenzonen, angewendet. Das Vermeiden von Kollisionen von Fahrzeugen untereinander ist eine weitere Funktion.

Unfälle im Untertagebereich, bei denen Personen von Maschinen überfahren oder gegen den Stoß gedrückt werden, sind mit zunehmender Mechanisierung leider sehr zahlreich.

In den Vereinigten Staaten von Amerika hat die Mine Safety and Health Administration (MSHA) die Unfälle seit $1984 \mathrm{im}$ Kohlenbergbau analysiert. Bei funkferngesteuerten Continuous Minern, bei denen der Maschinenfahrer mit dem Funkfernsteuerpult in der Nähe der Maschine steht, wurden für den Zeitraum Oktober 1984 bis September 2014 insgesamt 38 tödliche Unfälle gefunden. Dabei wurden die Verunglückten durch ungeplante Maschinenbewegungen und/oder durch Wahl des falschen Standortes von der Maschine an Stoß oder Firste eingeklemmt (Abb. 1; [1]).

Weiters hat MSHA bei der Analyse der Unfälle für mobile Transportmaschinen im Kohlenbergbau für den Zeitraum von 1984 und 2014 insgesamt 42 tödliche und 179 verletzende Unfälle festgestellt, welche bei Einsatz eines Schutzsystems zur Erkennung von Personen in Gefahrenzonen möglicherweise zu vermeiden gewesen wären [2].

Auch im Tunnelbau kommt es immer wieder zu solchen Unfällen, nur werden wegen der Kurzzeitigkeit der Baustellen weniger Unfallstatistiken erfasst und veröffentlicht. Die „International Tunnelling and Underground

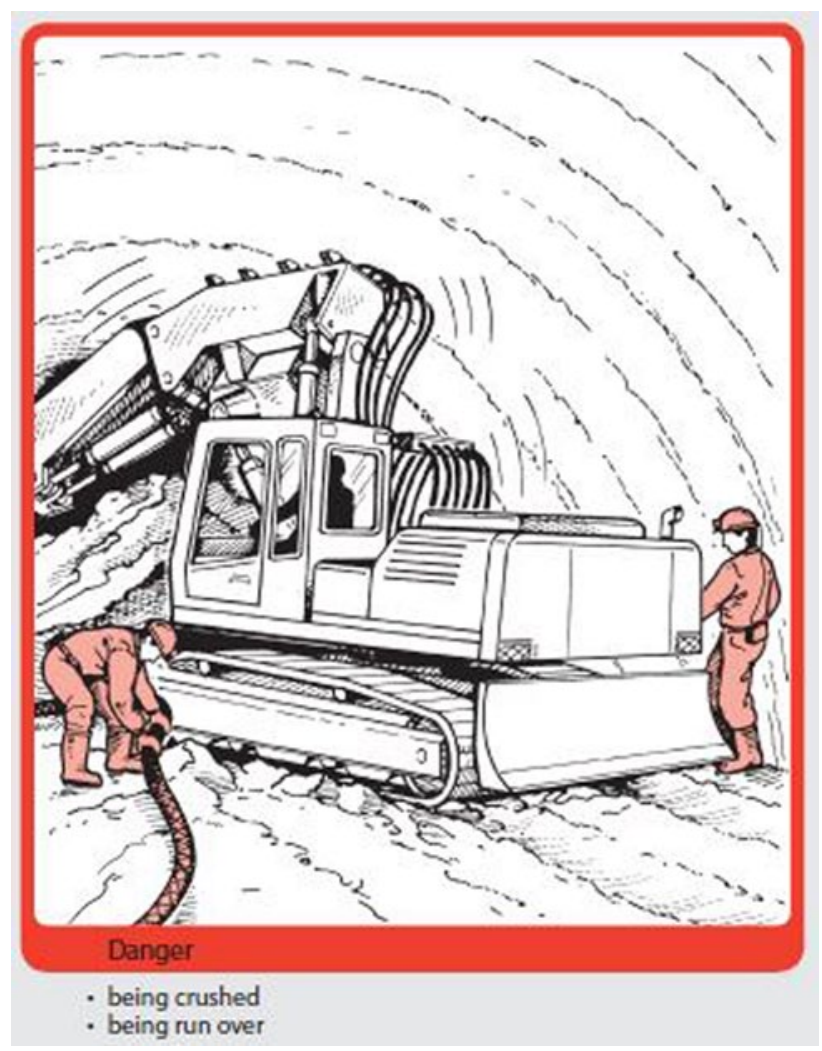

Abb. 2: Gefahren im Tunnelbau für Personen in der Nähe eines Baggers. Im toten Sichtwinkel des Baggerfahrers ist die Gefährdung, überfahren oder eingequetscht zu werden, besonders groß. (Quelle: ITA [3])

Space Association" hat die Richtlinie "Safe Working in Tunnelling" [3] veröffentlicht, in der auch die Gefahren für Personen in der Umgebung von mobilen Maschinen dargestellt werden. Besonders wird auf die Gefahr hingewiesen, von Transportfahrzeugen oder von Baggern oder von Teilschnittmaschinen erfasst und eingeklemmt zu werden. (Abb. 2)

In Österreich ereignete sich am 13. November 2015 an der BaustelleTunnelkette Granitztal bei St. Paul im Lavanttal ein tödlicher Arbeitsunfall, der in diese Kategorie fällt [4]. Ein 26-jähriger Arbeiter wollte gegen 23.15 Uhr an einem Bagger vorbeigehen. Als der Bagger nach rechts schwenkte, wurde der Arbeiter vom Baufahrzeug erfasst und gegen die Tunnelwand gedrückt. Dabei wurde der Mann so schwer verletzt, dass er noch an der Unfallstelle verstarb.

Auch in Europa, Australien und Südafrika sind solche Unfälle dokumentiert. Eine große Anzahl dieser Unfälle wäre durch Proximity Detection Systeme, die bei Anwesenheit einer Person in der roten Gefahrenzone die entsprechende gefährliche Maschinenbewegung stoppt, zu verhindern gewesen.

Alle diese Unfallberichte zeigen die Notwendigkeit von technischen Schutzsystemen, die Kollisionen zwischen Maschinen und von Menschen und Maschinen im Bergund Tunnelbau verhindern. 


\section{Technische Methoden und derzeit verfügbare Systeme}

Der derzeitige Stand der Arbeitstechnik untertage, bei dem der Mensch in enger Zusammenarbeit mit Maschinen den Vortrieb oder die Gewinnung durchführt, erfordert eine räumliche Nähe, die rasch in Gefahr umschlagen kann.

Die Abb. 3 dient zur allgemeinen Beschreibung eines technischen Schutzsystems gegen Kollision von Mensch und Maschinen in einer Untertagesituation. In diesem Beispiel wird um die Maschine ein elektromagnetisches Feld erzeugt, dessen Stärke von einem Empfänger an der Person (sogenanntes Poximity Detection Tag) gemessen und an die Maschine über RFID zurückgemeldet wird. Dabei wird die Entfernung zur Maschine berechnet und auch das Tag identifiziert. In der äußeren Zone wird die Anwesenheit einer Person erkannt, es erfolgt noch keine Aktion. In der mittleren Zone werden die Warnsysteme auf der Maschine und bei der Person aktiviert, welche die Nähe der Gefahrenzone anzeigen. Es können auch die Bewegungen der Maschine verlangsamt werden. In der inneren Zone, der unmittelbaren Gefahrenzone, werden die entsprechenden Maschinenbewegungen angehalten und die Gefahr signalisiert.

An technischen Methoden zur Erkennung von Personen in Gefahrenzonen untertage kommen zum Einsatz bzw. werden untersucht $[5,6]$ :

- Elektromagnetische Feldmessungen

- RADAR

- Radio Frequency Identification (RFID)

- Optische Videosysteme (3D-Vision)

- Thermobild-Kamerasysteme

- Schalllaufzeitmessungen

- Time-of-Flight Systeme
Die bisher im Untertageeinsatz befindlichen Systeme nützen eine Kombination von elektromagnetischen Feldmessungen und RFID-Kommunikation. Dabei sind ein oder mehrere Feldgenerator(en), je nach Größe und Form der Maschine, auf dem System, von dem die Gefahr ausgeht. Außerdem sind alle Personen im Untertagebereich mit einem Gerät ausgerüstet, Tag genannt, das die Feldstärke misst und über RFID an die Maschine zurückmeldet.

Die weitere Entwicklung erscheint besonders für RADAR basierende Systeme, Time-of-Flight Systeme und Kamerasysteme (optisch und infrarot) recht erfolgversprechend. Diese Systeme könnten auch eine Person im Gefahrenbereich ohneTag erkennen. Aber die erschwerten Umweltbedingungen untertage erfordern besondere Anpassungen (Abb. 4).

\section{Situation in den USA}

1998 begann auf Grund der zahlreichen Unfälle das "National Institute for Occupational Safety and Health" (NIOSH) mit der Entwicklung eines Schutzsystems für Personen in Gefahrenzonen um Bergbaumaschinen [7]. 1999 wurde ein entsprechendes Patent [8] erteilt, und die Firmen Geosteering Mining Services und Matrix Design Group nahmen Lizenzen und entwickelten bis 2009 Proximity Detection Systeme, die auch für den Einsatz im schlagwettergefährdeten Kohlenbergbau geeignet waren $([9,10] ;$ Abb. 5).

Wegen der vielen Unfälle in den Gefahrenzonen um Maschinen führte die MSHA in den Jahren 2004/2005 eine "Red Zone Awareness Campaign" durch, die durch Schulungsmaßnahmen auf die Gefahren hinwies. Dies brachte 2005 bis 2007 einen leichten Rückgang dieser Unfälle, dann stiegen die Unfallzahlen wieder an. Dadurch wurde MSHA überzeugt, dass technische Schutzmaß-

Abb. 3: Übersicht über ein technisches Schutzsystem, basierend auf der Kombination elektromagnetisches Feld/RFID. Die Person mit dem Tag Nr. 3 befindet sich innerhalb der Gefahrenzone des Laders LHD 1 und stoppt die Maschine. Die Person mit dem Tag Nr. 1 ist an der Grenze zur Warnungszone des Laders LHD 2 (Skizze: N. Sifferlinger)

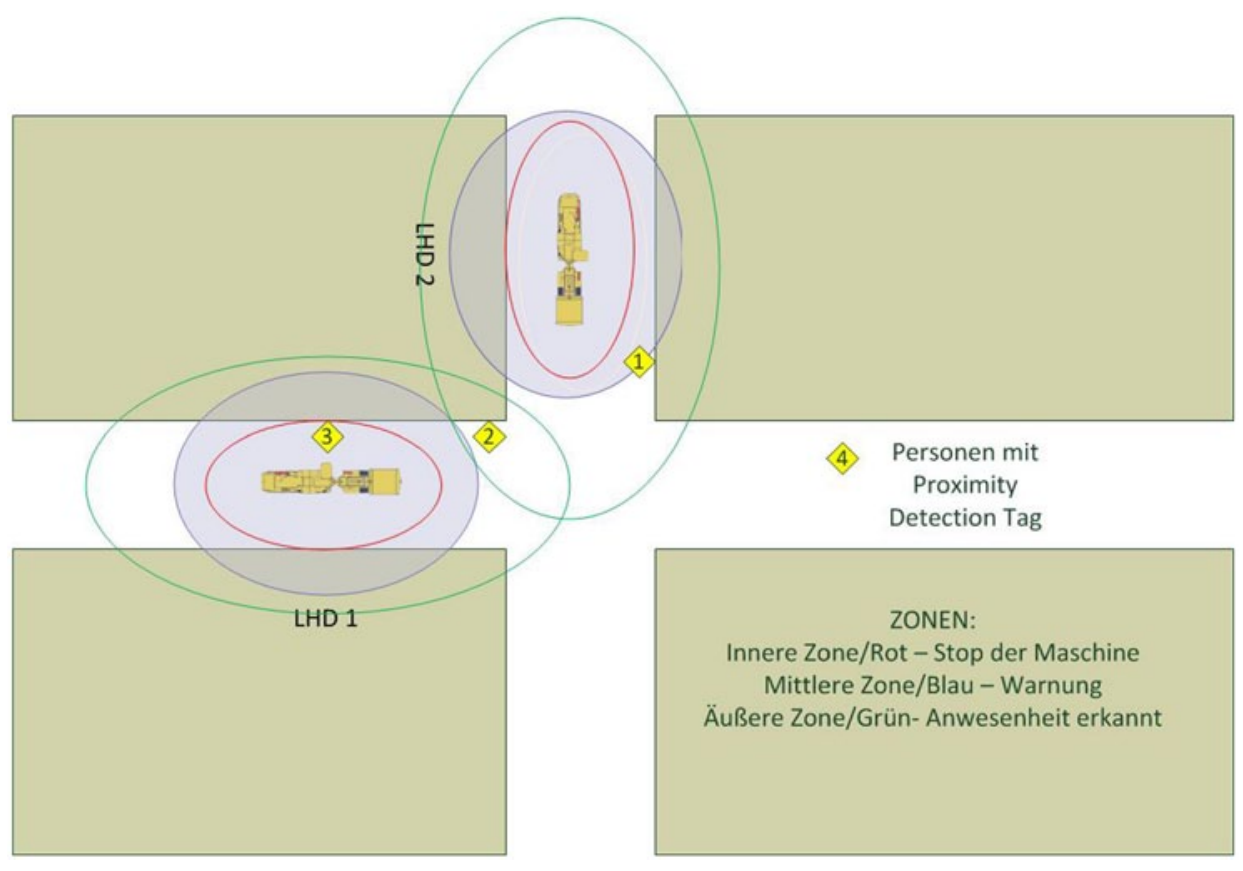




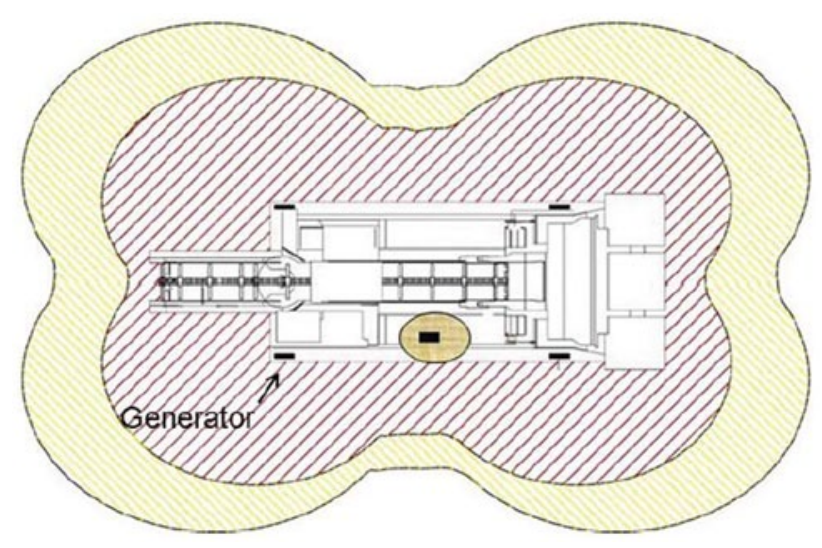

Abb. 4: Prinzip des elektromagnetischen Feldes um einen Continuous Miner, erzeugt von vier Generatoren auf der Maschine. Dabei ist zu beachten, dass das Feld in Form einer dreidimensionalen Blase um die Maschine liegt. (Quelle: NIOSH [5])

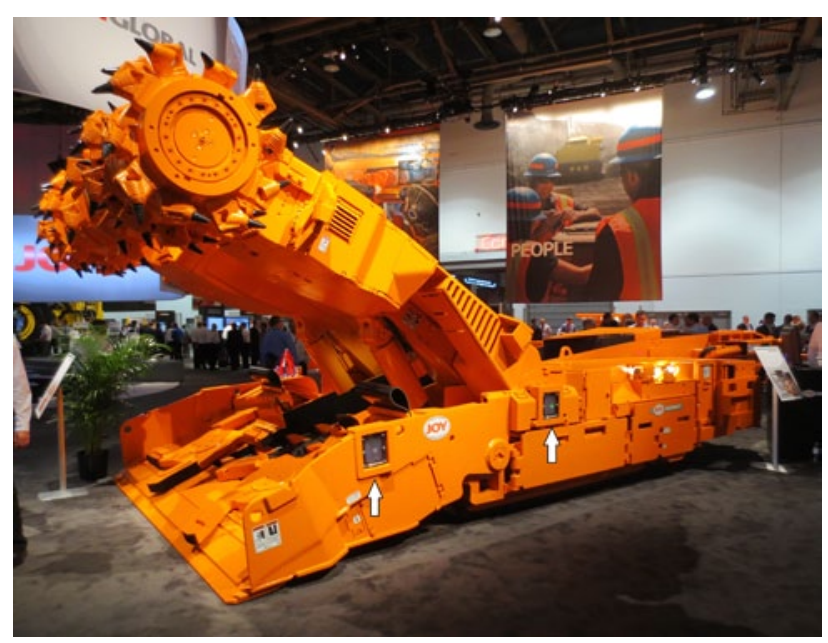

Abb. 6: JOY Continuous Miner 12CM mit dem Matrix M3-1000 Proximity Detection System, auf der Las Vegas Mining Show im September 2012. Die Feldgeneratoren bei diesem System sehen aus wie quadratische Fenster (Foto: N. Sifferlinger)

nahmen notwendig sind, und begann ein entsprechendes Programm mit der Industrie [11].

Am 15. Jänner 2015 publizierte die MSHA die endgültige Vorschrift für den Einsatz von Proximity Detection Systemen an Continuous Mining Maschinen [12]. Diese trat mit 16. März 2015 in Kraft und hat unter anderem folgenden Inhalt:

Continuous Mining Maschinen, welche nach dem 16. März 2015 hergestellt werden, müssen bis zum 16 . November 2015 mit einem Proximity Detection System ausgerüstet sein.

Continuous Mining Maschinen, welche vor dem 16. März 2015 hergestellt wurden und noch kein den Vorschriften entsprechendes Proximity Detection System haben, müssen bis zum 16. März 2018 mit einem vorschriftsmäßigen System ausgerüstet werden.

Ein vorschriftsmäßiges Proximity Detection System beinhaltet Komponenten auf der Maschine sowie Kom-

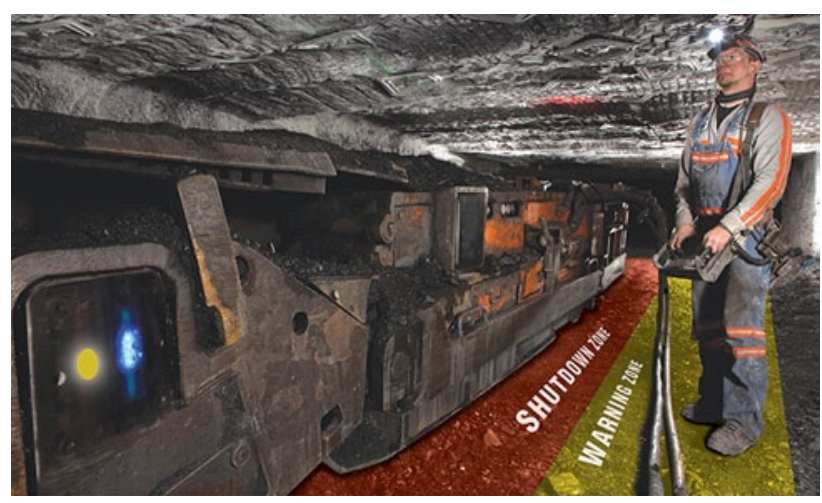

Abb. 5: Funkferngesteuerter Continuous Miner mit Matrix M3-1000 Proximity Detection System in den USA. (Quelle: Matrix [10])

ponenten, die vom Personal untertage getragen werden müssen, und hat folgenden Erfordernissen zu entsprechen:

1. Wenn eine Person in den Gefahrenbereich kommt, hat die Bewegung anzuhalten, bevor die Maschine in Kontakt mit der Person kommt.

2. Die Komponente, die von der Person getragen wird, muss ein optisches und ein akustisches Warnsignal abgeben, wenn die Person in die Warnungszone kommt, noch bevor die Gefahrenzone erreicht wird und die Maschine gestoppt wird. Auf der Maschine muss ein optisches Warnsignal den Maschinenfahrer über die Nähe einer Person informieren (Abb. 6).

Mit 25. November 2015 war im US-Kohlenbergbau nach Angaben von MSHA/NIOSH [13] die folgende Anzahl von Maschinen mit Proximity Detection Systemen ausgerüstet: Continuous Miner: 479; Shuttle Cars: 61; Coal Haulers: 33; Scoops: 63; Lademaschinen: 24;

Roof Bolter: 1; Feeder Breaker: 1; Sonstiges mobiles Gerät: 4; Gesamtsumme: 666 Systeme.

Im Jahre 2015 gab es im US-Kohlenbergbau rund 950 Continuous Miner, von denen infolge der Reduktion der Fördermengen nur rund 800 im Einsatz waren. Damit wurden bereits mehr als die Hälfte der Maschinen mit einem Proximity Detection System ausgerüstet.

MSHA listet auf der speziellen Seite für die Proximity Detection Technologie derzeit 14 Hersteller von solchen Systemen, davon derzeit vier mit Ex-Schutz-Zulassung für den Kohlenbergbau [9].

Im September 2015 kündigte MSHA an, auch für die mobilen Transportfahrzeuge im Untertage-Kohlenbergbau den Einsatz von Proximity Detection Systemen durch eine weitere Vorschrift zu verlangen [2]. Derzeit finden die öffentlichen Anhörungen sowie die Annahme von schriftlichen Stellungnahmen zu diesem Vorschriftsentwurf statt (die Kommentarfrist endete mit 1. Dezember 2015), und eine Ausweitung auf die mobilen Transportfahrzeuge ist für 2016 zu erwarten.

$\mathrm{NIOSH}$ beschäftigt sich in seinem Forschungszentrum in Pittsburgh weiterhin intensiv mit der Verbesserung der Proximity Detection Systeme, basierend auf den Einsatzerfahrungen im US-Kohlenbergbau und ausführlichen Messeinsätzen [14]. 


\section{Situation in Südafrika}

Im Frühjahr 2015 hat das Department of Mineral Resources für den Südafrikanischen Bergbau eine Ergänzung für die Vorschriften für Maschinen und Gerätschaften in Kraft gesetzt, welche für schienenungebundene mobile Maschinen Folgendes vorschreibt [15]:

Zur Vermeidung von Kollisionen zwischen schienenungebundenen mobilen Maschinen und Fußgängern müssen entsprechende Einrichtungen verwendet werden, die die Präsenz von Fußgängern in der Umgebung automatisch erkennen und im Gefahrenfall die Geschwindigkeit des Fahrzeuges automatisch bis zum Stillstand reduzieren.

Zur Vermeidung von Kollisionen zwischen dieselbetriebenen schienenungebundenen mobilen Maschinen untereinander müssen entsprechende Einrichtungen verwendet werden, die die Präsenz von anderen Maschinen in der Umgebung automatisch erkennen und im Gefahrenfall die Geschwindigkeit der beteiligten Maschinen, wenn die Maschinenfahrer auf die Warnungen nicht reagieren, automatisch bis zum Stillstand reduzieren.

Dies gilt sowohl für Obertage- wie auch Untertage-Betriebe.

Aber Südafrika war bereits ab etwa 2008 bei der Einführung der Proximity Detection Systeme weltweit führend. Für Shuttle Cars und Continuous Miner wurde von den großen Kohlenbergbaufirmen noch lange vor der gesetzlichen Pflicht mit der flächendeckenden Einführung dieser Systeme begonnen.

Valery Kononov, der sich bereits 1990 noch in der Sowjetunion in Donetsk mit möglichen Sicherheitssystemen beschäftigte [16], war nach seiner Auswanderung nach Südafrika im 21. Jahrhundert einer der Pioniere der neuen Technik [17]. Die südafrikanische Firma BOOYCO setzte die Ideen seinesTeams in ein erstes bergbautaugliches und für den Schlagwetterbergbau zugelassenes System um [18].

Aus Gesprächen mit südafrikanischen Bergbaubetreibern vor Ort ist dem Autor bekannt, dass die neuen Systeme schon vielfach vor ernsthaften Verletzungen bewahrt haben, dass es leider aber auch unerkannte Systemausfälle gegeben hat, die tödliche Unfälle dann nicht verhindern konnten. Daher ist der Wunsch nach einem System mit nachgewiesener Funktionaler Sicherheit gegeben. Allerdings haben die regionalen politischen und internationalen Turbulenzen durch die gesunkenen Rohstoffpreise entsprechende Entwicklungen durch Wegfall der wirtschaftlichen Deckung der Forschungs- und Entwicklungsbudgets in Südafrika im Moment fast zum Stillstand gebracht.

\section{Situation in Australien}

In Australien wurden seit dem Jahr 2010 der Bedarf und die Anforderungen an technische Schutzsysteme zur Erkennung von Personen in Gefahrenbereichen von Maschinen intensiv von den Bergbaubehörden und der Industrie diskutiert. So fand im Februar 2011 in Sydney ein "Proximity Detection and Collision Avoidance Systems Workshop" unter der Leitung der Bergbau- behörde von New South Wales statt, und im Februar 2014 wurde die Richtlinie "Mine Design Guideline MDG 2007 - Guideline for the selection and implementation of collision management systems for mining" von der Bergbehörde veröffentlicht [19]. 2013 fanden dann auch einzelne Erprobungen von Proximity Detection Systemen für den Untertagebergbau statt $[20,21]$, aber mit Beginn der Krise im Bergbau zur selben Zeit ist dann wohl aus Kostengründen von der Einführung solcher Systeme bisher Abstand genommen worden [22]. Auch die Diskussion, dass eigentlich nur ein System zum Einsatz kommen sollte, das den Anforderungen der Funktionalen Sicherheit entspricht - welches es aber noch nicht am Markt gibt - hat wohl die Verantwortlichen vor einer Beschaffung solcher Schutzsysteme zurückgehalten [23]. So nimmt der australische Bergbau, der sonst für sich in Anspruch nimmt, in Sicherheitsfragen der fortschrittlichste zu sein, hier eine abwartende Haltung ein.

\section{Situation in Europa}

Die europäische Typ C Norm EN 12111:2014 fürTunnelbaumaschinen und Continuous Miners - Sicherheitstechnische Anforderungen führt in der Liste der signifikanten Gefährdungen an [24]:

Mechanische Gefährdungen (wie Quetschen, Verwickeln, Einziehen); unerwartetes Anlaufen;

zusätzliche Gefährdungen aufgrund der Fahrfunktion der Maschine; zusätzliche Gefährdungen infolge Beweglichkeit in Bezug auf die Arbeitsposition der Maschine; zusätzliche Gefährdungen infolge Beweglichkeit in Bezug auf das Steuersystem; zusätzliche Gefährdungen infolge Beweglichkeit in Bezug auf dritte Personen; zusätzliche Gefährdungen infolge Arbeit unterTage.

Und zum Thema Schutzeinrichtungen: „Wo Personen arbeiten oder Zugang haben und ein Verletzungsrisiko durch Quetschen, Verwickeln, Fangen, Schneiden oder Erfassen durch drehende Teile an der Maschine besteht, müssen diese Teile durch Schutzeinrichtungen gesichert sein. Schutzeinrichtungen müssen mit EN 953 übereinstimmen. Diese Anforderung gilt nicht für den Schneidkopf und die Ladevorrichtung."

EN 953:2009 [25] definiert für die Sicherheit von Maschinen - Trennende Schutzeinrichtungen die allgemeinen Anforderungen an Gestaltung und Bau von feststehenden und beweglichen trennenden Schutzeinrichtungen. Es wird in der Norm ausdrücklich festgehalten, dass diese keine Anforderungen für besondere Schutzsysteme, die besonders für die Mobilität vorgesehen sind, enthält.

Die EN 16228-2:2014 für die Sicherheit von mobilen Bohrgeräten für Tiefbau, Geotechnik und Gewinnung von 15. November 2014 fordert einen eingeschränkten Zugang zu einem Gefahrenbereich mit drehenden Teilen [26]: „Bohrwagen müssen Bewegungsmelder haben, um den Zugang von Personen zum Gefahrenbereich mit drehenden Teilen zu melden", und "Wenn die Maschine in der automatischen Betriebsart für den vollen Bohrzyklus betrieben wird, muss das Bohren unterbrochen werden, 


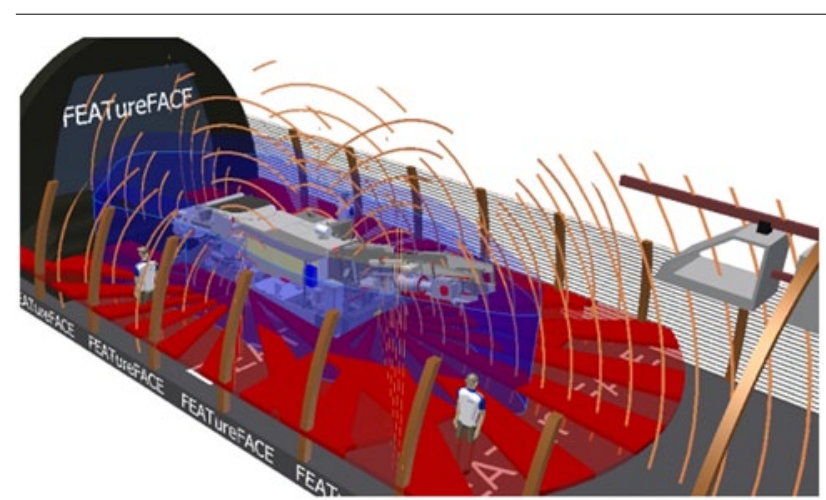

Abb. 7: Das prinzipielle Konzept einer $360^{\circ}$ Überwachung von FEATureFACE am Beispiel einerTeilschnittmaschine (Quelle: IMR/RWTH Aachen)

sobald eine Person den Bewegungsmelder passiert." Dazu die Anmerkung: „Es kann andere technische Lösungen als Bewegungsmelder geben, mit denen das gleiche oder ein besseres Schutzniveau erreicht werden kann. Dieses in Kombination mit den Felswänden begrenzt den Zugang zum Gefahrenbereich."

Diese Normen fordern noch kein explizites Proximity Detection System.

Die Europäische Union förderte unter der Projektnummer RFC CT-2012-00001 aus dem "Research Fund for Coal and Steel“ in den Jahren 2012 bis 2015 die Entwicklung von Technologien für ein Umfeldüberwachungssystem für den Untertagebergbau (Abb. 7).

Gemeinsam wurden in diesem Projekt vom Institut für Maschinentechnik der Rohstoffindustrie der RWTH Aachen, der Becker Mining $\mathrm{GmbH}$, der Indurad $\mathrm{GmbH}$ und der Linz Center of Mechatronics $\mathrm{GmbH}$ folgende Technologien adaptiert und erprobt [27]: Ultra-High-Frequency Radio Frequency Identification; niederfrequente magnetische Feldtechnologie; aktive und passive RADAR Technologien; Schalllaufzeitmessung zur Ortsbestimmung.

Dabei eignet sich die UHF-RFID Technologie besonders für größere Entfernungen bis 100 m, ist aber im Nahbereich zu ungenau. Das magnetische Feld ist für die Nahbereichsmessung bis zu $10 \mathrm{~m}$ sehr gut geeignet. Die RADAR Technologien und das Schalllaufzeitmesssystem eignen sich für den mittleren Bereich.

Es wurden bewusst physikalisch unterschiedliche Technologien gewählt, um damit die Grundlage für ein zukünftiges System mit Funktionaler Sicherheit zu schaffen.

\section{Erprobung und notwendige Tests}

Untertage wurden bis 2015 in Serienreife nur Systeme eingesetzt, welche die Schutzzone um die Maschine durch eine Kombination von elektromagnetischem Feld und RFID-Techniken erzeugen und überwachen. Dies ermöglicht eine Entfernungsmessung mit einer Genauigkeit auf Zentimeter.

Dabei ist es auch wichtig zu beachten, dass sich Magnetfelder mit selber Frequenz von verschieden Maschinen mit einem solchem System gegenseitig beeinflussen und zu gefährlichen Abweichungen führen können. Daher muss die Anwesenheit von weiteren Proximity Detection Systemen vor Ort erkannt und eine entsprechende synchronisierte zeitliche Taktung für eine gesicherte Abstandsmessung durchgeführt werden.

Dieser Sachverhalt ist auch bei Verwendung von Systemen verschiedener Hersteller kritisch, wenn sich ihre Felder nicht getaktet synchronisieren können und damit unplanmäßig gegenseitig stören. Dies ist auch ein wichtiger Punkt für die weitere Arbeit und Gesetzgebung der zuständigen Behörden.

Diese Proximity Detection Systeme auf Basis der magnetischen Feldstärke müssen während der Entwicklung, bei der Inbetriebnahme, bei regelmäßigen Wartungsarbeiten und nach Reparaturarbeiten auf die richtige Funktion überprüft werden. Dazu werden eigene Feldstärke-Messgeräte verwendet.

Die Testaufgaben für ein Proximity Detection System umfassen unter anderem folgende Punkte [28]:

- Feldstärke durch das Gestein/Kohle bei Querschlägen

- Einfluss anderer Maschinen in der Nähe, mit oder ohne Proximity Detection System

- Einfluss der Untertage-Infrastruktur, wie Rohre, metallene Gitter und Anker, Förderbänder

- Test desTag an der Person auf Funktion in verschiedenen Höhen: Schuh-, Gürtel-, Helmhöhe

- Test auf Wiederholbarkeit und Konsistenz der Erkennung der Gefahrenzonen (-Grenzen)

- Nahbereich-Überprüfung

- Einfluss verschiedener Maschinenstellungen (bei LHD z. B. Schaufel angehoben/abgesenkt oder links/mitte/ rechts)

- mögliche Fehler/Missbrauchsszenarien wie Metall nahe dem Tag oder den Antennen.

Besonders die Beeinflussung des Magnetfeldes durch Metallteile im Nahbereich kann gefährlich werden - so kann zum Beispiel ein Tag am Gürtel durch eine Werkzeugtasche in der Nähe falsche Abstandsresultate liefern.

\section{Erfahrungen aus dem Betrieb bestehender Systeme}

Das National Institute for Occupational Safety and Health hat sich intensiv mit der Evaluierung der verfügbaren Proximity Detection Systeme für Continuous Miner in den Vereinigten Staaten beschäftigt [29, 30].

Für Schutzsysteme zur Erkennung von Personen im Gefahrenbereich sind zwei Arten von Fehlern besonders kritisch:

a) Nichterkennen einer Person im Gefahrenbereich

- Ausfall des Systems

- kein Tag an der zu schützenden Person

- Verfälschung der Abstandsmessung 
Abb. 8: Blockdiagramm eines möglichen „Proximity Detection Systems mit Funktionaler Sicherheit." Diese wird unter anderem durch Redundanz, Selbstdiagnosefunktionen und Hardware in SIL-Ausführung erreicht. (Skizze: N. Sifferlinger)

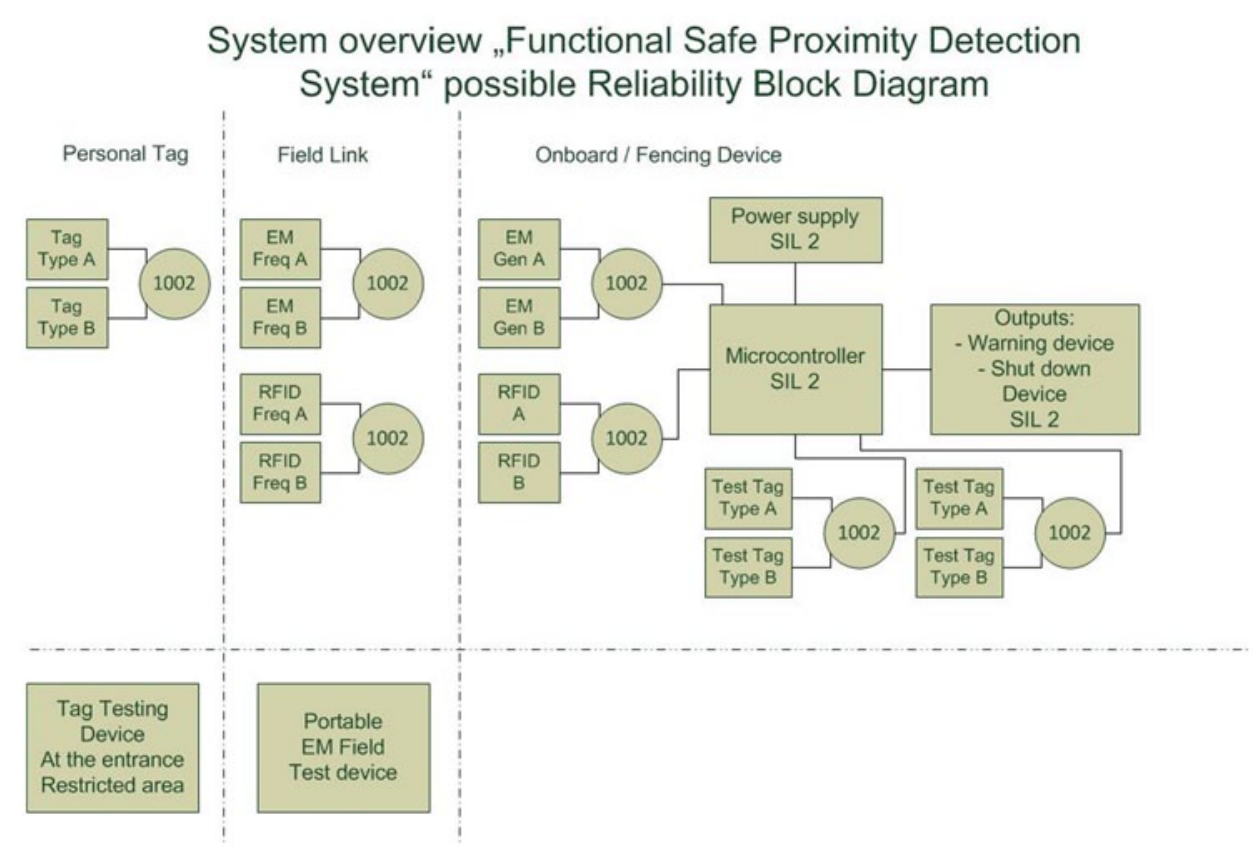

System overview „Functional Safe Proximity Detection System“ possible Reliability Block Diagram

[31], lässt sich die Sicherheitsintegrität der Hard- und Software erhöhen. Damit steigt die Wahrscheinlichkeit, dass die sicherheitsbezogenen Steuerungsfunktionen unter allen festgelegten Bedingungen zufriedenstellend ausgeführt werden.

Derzeit ist es im Bergbau so, dass Bereiche, in denen autonome, unbemannte Fahrzeuge unterwegs sind, vollkommen für Personen abgesperrt werden müssen. Mit einem Umfeldüberwachungssystem, welches den Anforderungen der Funktionalen Sicherheit entspricht, könnte eine Koexistenz möglich werden (Abb. 8).

\section{Ausblick}

Die Weiterentwicklung der Schutzsysteme gegen Kollision von Mensch und Maschine im Untertagebereich ist im Gange, mit nachgewiesener Betriebszuverlässigkeit werden auch die Behörden die Vorschriften entsprechend nachschärfen. Damit wird die Anwendung dieser Schutzeinrichtungen im Bergbau - aber auch im Tunnelbau - zum Standard werden.

Open Access Dieser Artikel unterliegt den Bedingungen der Creative Commons Attribution License. Dadurch sind die Nutzung, Verteilung und Reproduktion erlaubt, sofern der/die Originalautor/en und die Quelle angegeben sind.

\section{Literatur}

1. Huntley, C.; Chirdon, D.: Remote Controlled Continuous Mining Machine Fatal Accident Analysis Report of Victim's Physical Location with Respect to the Machine; U.S. Department of Labor, Mine Safety and Health Administration (MSHA) 2014, http://www. msha.gov/webcasts/Coal2005/Fatal\%20Accident\%20Summary. pdf (13.12.2015) 
2. MSHA: Proposed Rule for Proximity Detection Systems for Mobile Machines in Underground Coal Mines to Prevent Deaths and Injuries, U.S. Department of Labor, Mine Safety and Health Administration (MSHA) 2015, http://www.msha.gov/REGS/ FEDREG/PROPOSED/2015/proximity-detection-mobile/

3. Safe Working in Tunnelling, ITA Working Group Health and Safety, http://www.ita-aites.org/en/future-events/89-safe-working-intunnelling-2011 (13.12.2015)

4. Kleine Zeitung: Bagger erdrückt 26-Jährigen bei Tunnelarbeiten, http://www.kleinezeitung.at/k/kärnten/lavanttal/4866329/ (14.11.2015)

5. Li, J.; Carr, J.; Jober, C.: A shell-based magnetic field model for magnetic proximity detection systems, The National Institute for Occupational Safety and Health, http://www.cdc.gov/niosh/ mining/UserFiles/works/pdfs/asbmf.pdf (14.12.2015)

6. NIOSH: Engineering Considerations and Selection Criteria for Proximity Warning Systems for Mining Operations, Centers for Disease Control and Prevention, NIOSH, September 19, 2012, http://www.cdc.gov/niosh/mining/content/pwsselection.html (13.12.2015)

7. Ruff, T:: Overview of Proximity Warning Technology and Approaches, Presentation, NIOSH Workshop on Proximity Warning Systems, Charleston, West Virginia, September 15, 20102010

8. United States Patent US 5.939.986, Mobile Machine Hazardous Working Zone Warning System, Schiffbauer et al., Date of Patent August 17, 1999

9. MSHA: Proximity Detection/Collision Warning Information from Technical Support, http://www.msha.gov/Accident_Prevention/ NewTechnologies/ProximityDetection/ (06.12.2015)

10. Matrix: M3-1000 Proximity Detection, http://www.matrixteam. com/products/m3-1000.htm (12.12.2015)

11. Chirdon, D.: MSHA Proximity Detection, Approval and Certification Center, Mine Safety and Health Administration (MSHA), February 2, 2009, Document MSHA-2014-0019-0011

12. MSHA: Proximity Detection Systems for Continuous Mining Machines in Underground Coal Mines, Final rule effective March 16, 2015, U.S. Department of Labor, Mine Safety and Health Administration (MSHA), http://www.msha.gov/regs/fedreg/ final/2015/proximity-detection/ (13.12.2015)

13. E-Mail von David Chirdon/CDC/NIOSH an den Autor am 25 . November 2015

14. Ducarme, J.; Carr, J.; Reyes, M.: Smart sensing: the next generation, Mining Magazine July/August 2013, p. 58-66

15. Department of Mineral Resources, No. R. 125, 27 February 2015, Mine Health and Safety Act, 1996 (ACT No 29 of 1996), Regulations relating to machinery and equipment, Schedule Regulation Amendments, Chapter 8, Trackless Mobile Machinery, Government Gazette No. 38493, 27 February 2015

16. World Intellectual Property Organisation; WO 90/10140, Device for manipulation control of a moveable object, International Application Published under the Patent Cooperation Treaty (PCT), International Publication Date 7 September 1990

17. World Intellectual Property Organisation; WO 2004/1090830 A1, Collision Avoidance Method and System, International Application Published under the Patent Cooperation Treaty (PCT), International Publication Date 21 October 2004
18. Lourens, A.: Collision avoidance, International Mining, September 2011, p. 58-59

19. New South Wales Government, Trade \& Investment, Mine Safety: MDG 2007 Guideline for the selection and implementation of collision management systems for mining, February 2014

20. Herbert, P. (Senior Inspector of Mines-Electrical): Proximity Detection Systems, Department of Natural Resources and Mines, Queensland Government 2013, http://www.resourcesandenergy. nsw.gov.au/_data/assets/pdf_file/0011/448166/Peter-HerbertProximity-Detection.pdf

21. Kent, D.; Schiffbauer, B.: Proximity Detection Systems in Underground Mines, http://www.qrc.org.au/conference/_dbase_upl/ kent\%20-\%20Proximity\%20Detection.pdf (12.12.2015)

22. Macdonald, C.: Underground lags surface in collision avoidance, Australian Journal of Mining, September/October 2013, p. 6-8

23. Engineers Australia: Electrical Presentation on Mine Functional Safety \& Proximity Detection in Underground Coal Mines, 15 October 2014, https://www.engineersaustralia.org.au/portal/ news/electrical-presentation-mine-functional-safety-proximitydetection-underground-coal-mines (13.12.2015)

24. ÖNORM: EN 12111, Tunnelbaumaschinen - Teilschnittmaschinen und Continuous Miners - Sicherheitstechnische Anforderungen, Ausgabe 2014-10-15

25. ÖNORM: EN 953, Sicherheit von Maschinen - Trennende Schutzeinrichtungen - Allgemeine Anforderungen an Gestaltung und Bau von feststehenden und beweglichen trennenden Schutzeinrichtungen, Ausgabe 2009-06-01

26. ÖNORM: EN 16228-2, Geräte für Bohr- und Gründungsarbeiten Sicherheit, Teil 2: Mobile Bohrgeräte für Tiefbau, Geotechnik und Gewinnung, Ausgabe 2014-11-15

27. Neumann, K.; Berg, J.; Eichentopf, B.; Mehnert, P.; Nienhaus, K.; Sauter, T.; Rabel, M.; Hammer, F; Pichler, M: FEATureFACE - an Innovative Collision Avoidance System for the Underground Mining Industry, in: Application of computers and operations research in the mineral industry: proceedings of the 37th International Symposium: APCOM 2015, p. 767-784

28. InfoTronix Collision Avoidance System, Underground Coal System Validation, Test Report, 4 September 2013, InfoTronix Document, Seven Hills, NSW, 2013.

29. Arbeitsgespräch von N. Sifferlinger mit J. Carr am 15. Juni 2015 bei NIOSH, Pittsburgh zum Thema „Evaluierung und Weiterentwicklung von Proximity Detection Systemen"

30. Jobes, C.; Carr, J.; DuCarme, J.: Evaluation of an Advanced Proximity Detection System for Continuous Mining Machines, The National Institute for Occupational Safety and Health, March 2012, http://www.cdc.gov/niosh/mining/UserFiles/works/pdfs/eoaap.pdf (13.12.2015)

31. ÖNORM: EN62061, Sicherheit von Maschinen - Funktionale Sicherheit sicherheitsbezogener elektrischer, elektronischer und programmierbarer elektronischer Steuerungen, Ausgabe 2013-10-01 